\title{
Corrected calculation of the overestimated ejection fraction in valvular heart disease by phase-contrast cardiac magnetic resonance imaging for better prediction of patient morbidity
}

Hosam A. Yousef ${ }^{1}$, Abdulrahman E. S. Hamdan ${ }^{1 *}$, Ahmed Elminshawy ${ }^{2}$, Nisreen A. A. Mohammed ${ }^{1}$ and Ahmed S. Ibrahim ${ }^{3}$

\begin{abstract}
Background: To establish a more accurate technique for the assessment of the left ventricular function correlated with patients' clinical condition avoiding the miscalculation of the ejection fraction in valvular regurgitation. A prospective study carried out between July 2018 and June 2019. The studied group included 35 subjects, 25 patients with valvular regurgitation, and 10 healthy control subjects. All subjects underwent cardiovascular magnetic resonance examination to evaluate the ejection fraction by two methods: the volumetric method which assesses stroke volume via subtraction of the end-systolic volume from the end-diastolic volume, and phasecontrast method which assesses the aortic stroke volume via a through-plane phase contrast across the aortic valve. The sensitivity, specificity, $P$ value and the area under the curve of both methods were calculated.

Results: In the healthy group, using the volumetric method, the calculated mean ejection fraction was $62.44 \pm 6.61$, while that calculated by the phase-contrast method was $64.34 \pm 5.33$, with a non-significant difference $(P=0.62)$ showing the validity of the phase-contrast method. In the patients' group, by using the volumetric method, the calculated mean ejection fraction was $47.17 \pm 14.31 \%$, which was significantly higher than that calculated by the phase-contrast method $(29.39 \pm 7.98 \%)(P=0.02)$. According to the results of the calculation of the ejection fraction by the volumetric method, there were 18 patients (72\%) having impaired cardiac function and 7 (28\%) patients of normal function; while according to the phase-contrast method, all the 25 patients had impaired cardiac function. The current study shows that the phase-contrast cardiac magnetic resonance had $89.29 \%$ sensitivity and $85.7 \%$ specificity in diagnosing impaired cardiac function with the area under the curve of $0.87(P=0.00)$.
\end{abstract}

Conclusion: The phase-contrast cardiac magnetic resonance can provide a better assessment of the ejection fraction in valvular regurgitation.

Keywords: Cardiac magnetic resonance imaging (CMR), Phase contrast (PC), Ejection fraction (EF), Valvular heart diseases (VHD), Regurgitation

\footnotetext{
* Correspondence: abdulrahman.emam@med.aun.edu.eg

${ }^{1}$ Radiology Department, Faculty of Medicine, Assiut University, Assiut, Egypt

Full list of author information is available at the end of the article
} 


\section{Background}

Accurate and reproducible assessment of the left ventricular (LV) volumes, in particular, the ejection fraction (EF), is important in the management of various cardiac diseases because it is one of the strongest predictors of the clinical outcome $[1,2]$.

Cardiac magnetic resonance (CMR) imaging has been established in the evaluation of different cardiac diseases, with proven high accuracy and reproducibility. CMR is considered a gold standard in the evaluation of LV function and is the recommended method to assess cardiac function and hemodynamics [3, 4], with higher reproducibility than echocardiography [5-7].

Phase-contrast (PC) velocity mapping shows a higher sensitivity to flow disturbances and can directly measure forward, regurgitant, and shunt flows in congenital and acquired heart disease $[8,9]$. Phase-contrast cardiac magnetic resonance (PC-CMR) techniques have gained popularity with improvements in MR hardware, pulse sequences, and post-processing techniques [10].

Previous studies have shown that PC-CMR provides the most accurate measurement of cardiac output and LV volumes in cases with mitral, aortic, or pulmonary valve regurgitation [11-14].

Valvular heart diseases (VHD) are common, and their incidence increases with age. In the past, VHD were typically caused by rheumatic heart diseases (RHD), which remain a significant public health burden in developing countries. In industrialized countries, rheumatic valve diseases have fallen so much and the risk of developing age-related degenerative valvular heart diseases increased as a result of increasing life expectancy and atherosclerotic risk factors [15].

In routine clinical practice, left ventricular performance is one of the most important prognostic factors in valvular heart diseases, whether treated medically or surgically. The left ventricular ejection fraction (LVEF) is the most commonly used clinical measure for the assessment of the global left ventricular function. It gives valuable information that may be useful in the selection of therapy or determination of the optimal time for intervention $[16,17]$.

The ejection fraction $(\mathrm{EF})$ is defined as the percentage of blood leaving the heart each time it contracts, and it is calculated through dividing the ventricular stroke volume [the difference between the end-diastolic volume (EDV) and end-systolic volume (ESV)] by the ventricular EDV, depending on the rule of summation of discs (Simpson's method) [18], using the following equation:

$$
\mathrm{LVEF}=100 \times \frac{\mathrm{EDV}-\mathrm{ESV}}{\mathrm{EDV}}
$$

This method is applied by most or even all modalities which are used to estimate the EF, either subjectively by visual estimation or objectively by quantitative methods, invasively or non-invasively, like echocardiography, magnetic resonance imaging (MRI), computed tomography (CT), gated equilibrium radionuclide angiography and gated myocardial perfusion imaging with either single-

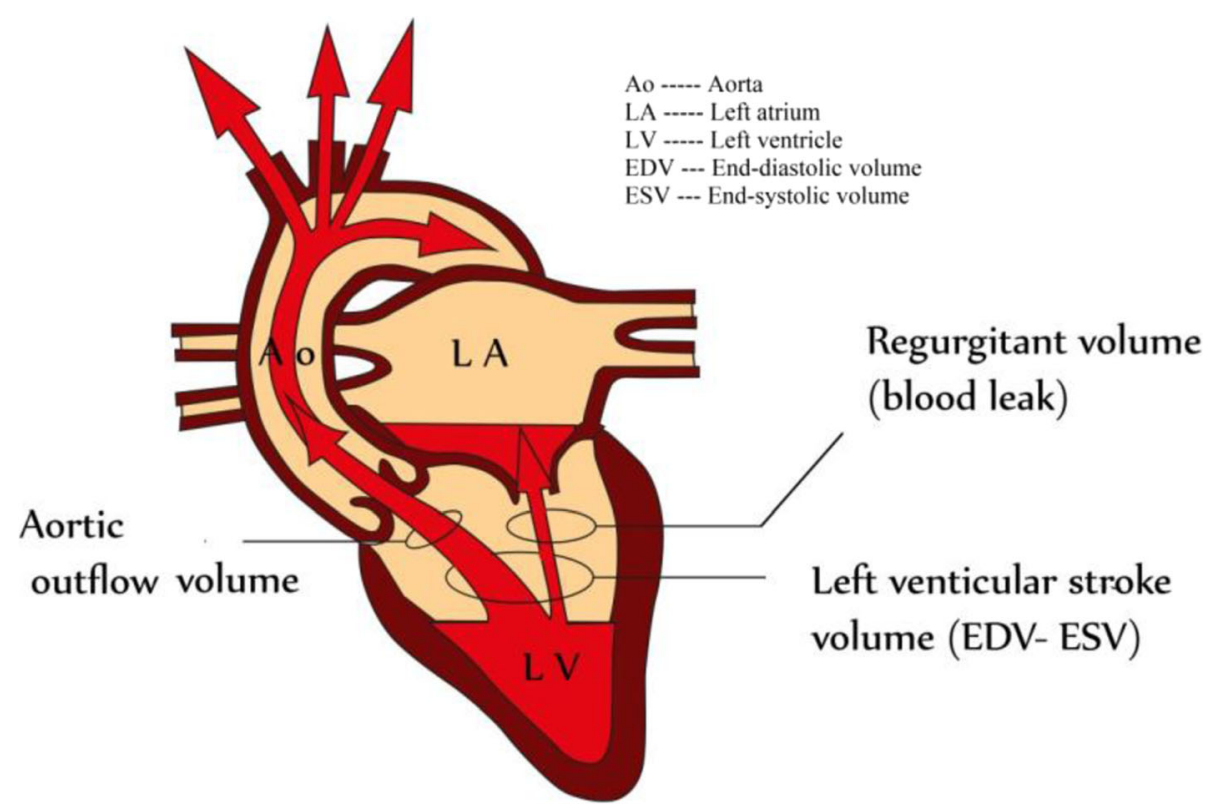

Fig. 1 In mitral valve regurgitation, the blood leaks backward during systole through the mitral valve into the left atrium dividing the stroke volume estimated by volumetric method into two components: the aortic flow volume which represent the effective stroke volume, and the regurgitant flow volume which still present within the heart overestimating the calculated ejection fraction 


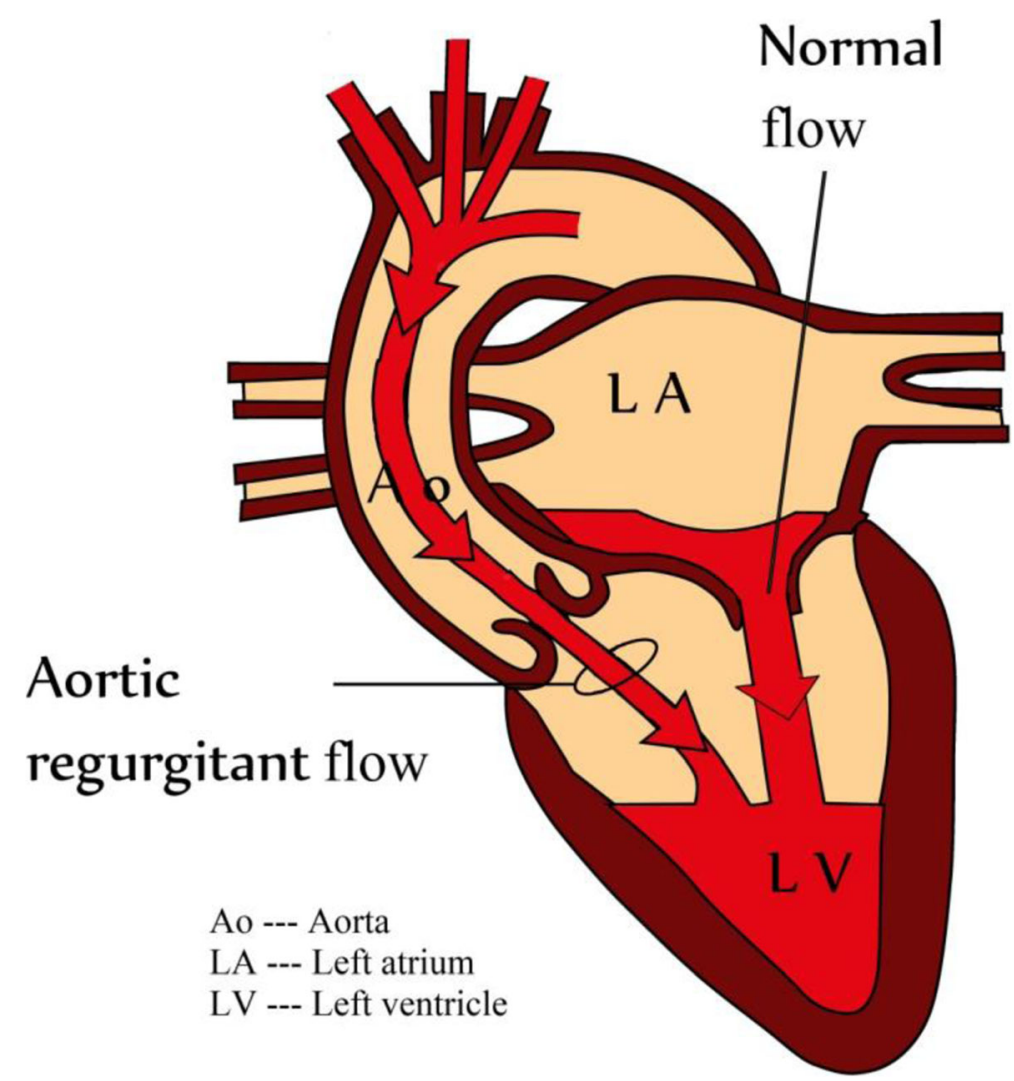

Fig. $\mathbf{2}$ In aortic valve regurgitation. The valve does not close completely during diastole with leaking of part of the ejected blood backward into the heart reducing the net ejection fraction throughout the whole cardiac cycle

photon emission computed tomography (SPECT) or positron emission tomography (PET) [19].

The ejection fraction represents the change in LV volume from end-diastole to end-systole without taking into consideration the direction of pumped blood. It estimates the amount of blood pumped out of the left ventricle wherever the blood is pumped through the aorta or through another leakage pathway [20].

In a heart with normally functioning valves, the blood volume entering the left ventricle across the mitral valve will be equal to the blood volume exiting the left ventricle across the aortic valve. On the other hand, in patients with valvular regurgitation, the LV stroke volume estimated by the standard method is equal to the aortic forward stroke volume crossing the aortic valve (antegrade flow), in addition to the blood volume that leaks through the diseased valve (retrograde flow). Actually, there are two possible conditions with regurgitation:

1) In the case of mitral regurgitation: the LVEF is overestimated because it does not only reflect the blood volume pumped into the aorta, but also that is pumped into the low-pressure left atrium [21]. As a result, the left ventricular stroke volume is actually divided into two components, the aortic forward flow volume and the regurgitant volume into the left atrium (Fig. 1). According to the standard method of calculation of the LVEF, the regurgitant blood volume is calculated as a part of the SV instead of subtracting it as it is not actually pumped outside the heart. This overestimation of the LV systolic function gives a false better indication of left ventricular function, while the patient may be clinically complaining or even in a situation of heart failure.

2) In the case of aortic regurgitation: the heart pumps blood out of the left ventricle during systole, and then a part of this pumped volume leaks back through the incompetent aortic valve during

Table 1 Normal ranges and severity partition cut-off values for LVEF

\begin{tabular}{lll}
\hline & Male & Female \\
\hline Normal range & $52-72$ & $54-74$ \\
Mildly abnormal & $41-51$ & $41-53$ \\
Moderately abnormal & $30-40$ & $30-40$ \\
Severely abnormal & $<30$ & $<30$ \\
\hline
\end{tabular}


Table 2 Diagnostic accuracy of phase contrast cardiac MRI in diagnosing impaired cardiac function

\begin{tabular}{ll}
\hline Indices & Value \\
\hline Area under the curve & 0.87 \\
Sensitivity & $89.29 \%$ \\
Specificity & $85.71 \%$ \\
Positive predictive value & $96 \%$ \\
Negative predictive value & $67 \%$ \\
$P$ value & 0.00 \\
\hline
\end{tabular}

diastole. When this happens, the net SV across the aortic valve is reduced (Fig. 2). So, again, according to the standard method for calculation of LVEF, the net aortic stroke volume is not actually calculated because a portion of the estimated pumped blood still remains in the left ventricle. This in turn overestimates the LVEF and gives a false better indication of the left ventricular function while the patient could be complaining or have a picture of heart failure.

So, we assume that, in patients with valvular regurgitation, the volumetric CMR method for estimation of the LVEF using the EDV and ESV may overestimate and miscalculate the indices of the left ventricular function, because the leaking regurgitant blood volume is not subtracted as it should be. There is actually a real need for a method that could correctly calculate the aortic SV that would determine the appropriate management of patients with valvular heart disease. In order to achieve the exact estimation of the aortic SV and in turn the LVEF, it is needed to calculate the blood volume pumped across the aortic valve throughout the whole cardiac cycle. By this method, the regurgitant blood volume across the malfunctioned valves would be subtracted.

Continuous improvements in MRI hardware and software have enabled MRI to increasingly attain an important role in a large variety of cardiac diseases, offering unique information including the ability to directly quantify flow using through-plane phase-contrast (PC) velocity mapping [22].

The aim of this study was to compare the standard volumetry CMR and PC-CMR techniques for estimation of the LVEF in patients with valvular heart diseases.

\section{Methods}

The current study is a prospective study comparing two methods of calculation of EF, the volumetric and PC methods, in valvular regurgitation. The institutional review board approved the study. The participants were recruited from the cardiothoracic department and

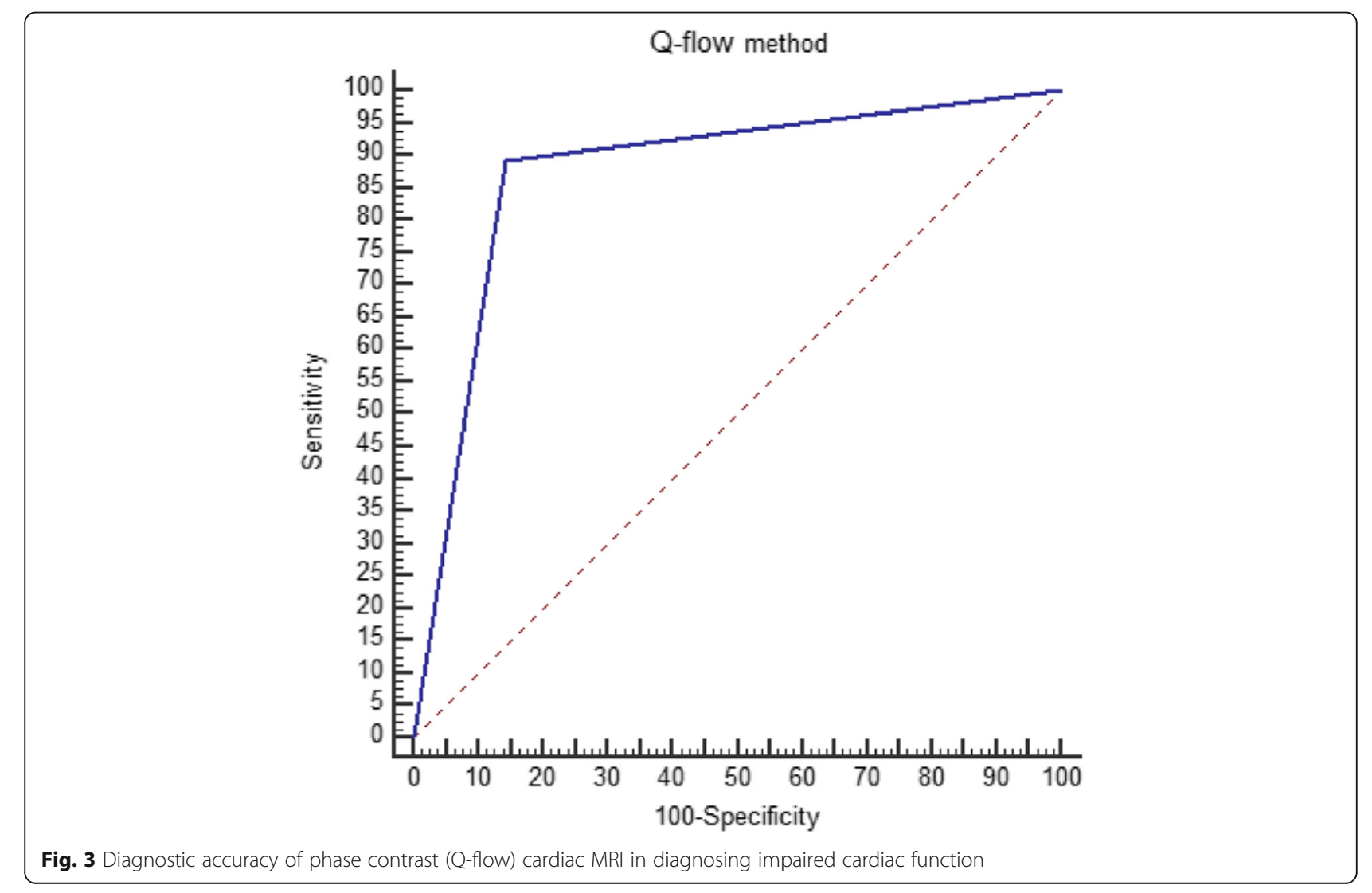



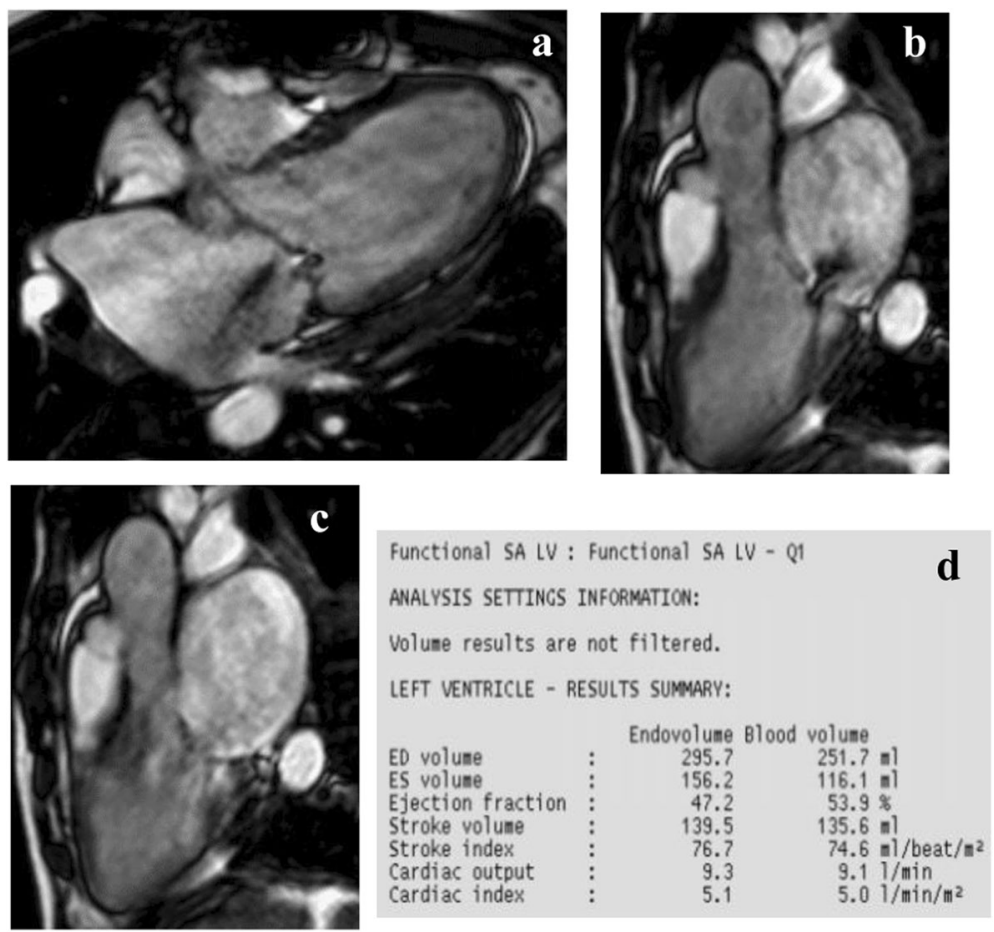

Fig. 4 A 51-year-old male patient with rheumatic valvular heart disease presented with dyspnea and lower limb edema. The performed CMR showed mitral and aortic regurge. Mitral regurge is seen in $\mathbf{a}$ four-chamber view during systole and $\mathbf{b}$ three-chamber view during systole, and aortic regurge is seen in $\mathbf{c}$ three-chamber view during diastole; $\mathbf{d}$ the volumetric analysis shows that ejection fraction estimated by volumetric short-axis method (EFvol) was $47.2 \%$ (mild dysfunction)

underwent CMR examination on a 1.5 Tesla system in the radiology department at a tertiary hospital in the period between July 2018 and June 2019.

\section{Study population}

The study was conducted on 35 subjects including 25 adult patients with reversed cardiac hemodynamics (aortic and mitral valve regurgitation) and 10 age- and sex-matched healthy controls with no clinically or echocardiographically detected cardiac abnormalities. The 25 patients have been diagnosed clinically and by echocardiography as having valvular heart disease (VHD) of different etiologies (14 rheumatic, 5 ischemic, 6 non-ischemic cardiomyopathic). Patients with known contraindication to MRI including the presence of paramagnetic surgical clips, pacemakers, severely ill patients, claustrophobics, restless subjects, and patients with arrhythmia were excluded.

\section{Image acquisition}

A standard CMR protocol was applied in all participants in the study including the following steps and pulse sequences: First of all, FFE multi-planar localizer for planning of the imaging views. Then functional cine images acquired using ECG-gated segmented k-space breath-hold balanced turbo field echo (b-TFE) sequence in short-axis view; two-, three- and four-chamber views; and left ventricular outflow tract (LVOT) view, all were obtained during repeated breath-hold. After that, volumetric images were acquired using a stack of 9 contiguous $8 \mathrm{~mm}$ slices in the double-oblique LV short-axis orientation, covering the whole LV from the apex to just above the level of the base of the heart. Lastly, the transaortic flow (Q-flow) was determined from through-plane phase-contrast images obtained with retrospective ECG synchronization during breath-hold. A velocity of 150 $\mathrm{cm} / \mathrm{s}$ was chosen for velocity encoding, and the phase encoding velocity was increased if aliasing was noticed. To define the acquisition plane, the three-chamber LVOT and coronal LVOT views were used during systole. The section was positioned just above the opened aortic valve at the sino-tubular portion. In order to minimize the effect of factors that may affect EF such as changes in heart rate, blood pressure, and resting conditions, we will compare the two methods by MRI examination which is performed at the same time on the same table of the MRI scanner.

\section{Images analysis}

All the obtained MR images were transferred to a computer workstation for analysis and post-processing. 

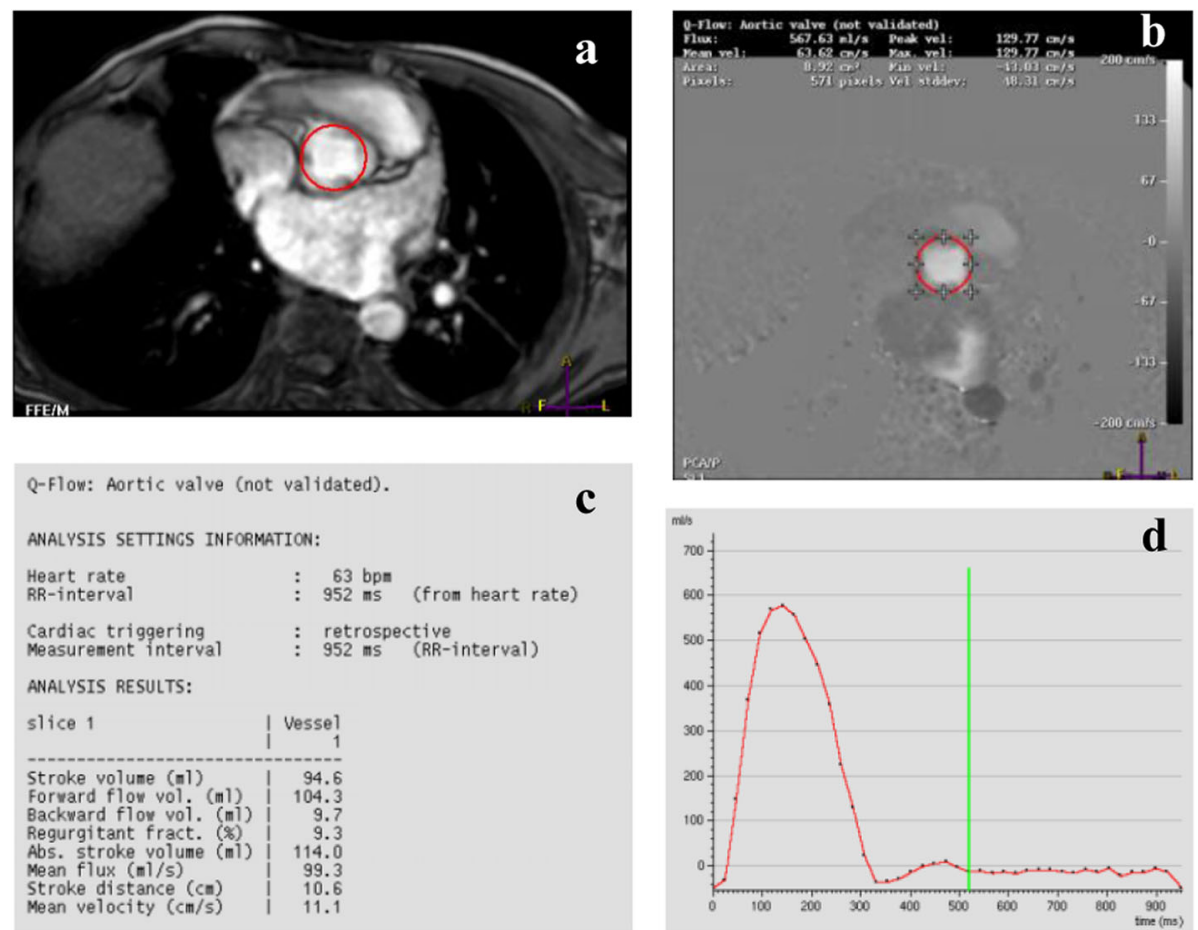

Fig. 5 On the other hand, the same patient using the phase-contrast analysis, the LV stroke volume (SVPC) was $94.6 \mathrm{ml}$ and ejection fraction $($ EFPC) was $(94.6 / 295.7) \times 100=32 \%$ which reflects moderate dysfunction. a The magnitute image showing the detailed anatomy, contour, and the shape of the aortic valve during systole. $\mathbf{b}$ The phase velocity map depicts the velocity and direction of flow in each pixel within the aorta. $\mathbf{c}$ Q-flow analysis of the aortic flow. $\mathbf{d}$ By outlining the contours of the aorta throughout each phase in the cardiac cycle, a flow curve can be generated to determine aortic forward reverse stroke volume and flow

Assessment of the LVSV and EF from the volumetric short-axis images (the volumetric method) was via measurement of LV EDV and ESV. Endocardial borders at the end-diastole and end-systole were manually traced using standard system software analysis tools. Volumes were computed by a summation of the discs method "Simpson's rule" where the sum of all slices was multiplied by the slice thickness. Subsequently, SV and EF were calculated using the following equation: "LVEF = SV(EDV - ESV) $\div$ EDV."

Whereas assessment of the LV SV and EF derived from the trans-aortic flow volume (the phase-contrast method) was via velocity maps which used to determine flow volume throughout the whole cardiac cycle, with the same magnitude and phase velocity maps, a region of interest was traced around the ascending aorta to determine the area of flow, frame by frame. By multiplying the velocity $(\mathrm{cm} / \mathrm{s})$ of each pixel by the area $\left(\mathrm{cm}^{2}\right)$ of the region of interest, the instantaneous flow volume $\left(\mathrm{cm}^{3} / \mathrm{s}\right)$ is obtained for each frame of the cardiac cycle. The instantaneous flow volume of each frame ( $y$-axis) can be plotted against the time of the cardiac cycle ( $x$-axis) to show bulk flow as it relates to the cardiac cycle. When the area under the curve was integrated for systole and diastole, forward, regurgitant and stroke volumes could be generated [23]. Subsequently, the EF was calculated via this equation: $\mathrm{LVEF}=$ aortic $\mathrm{SV}$ (phase contrast) $\div$ EDV.

The data obtained from both methods were classified and qualified according to the guidelines and recommendations of the American Society of Echocardiography (ASE) and the European Association of Cardiovascular Imaging (EACVI) 2015 [24] as shown in Table 1.

\section{Statistical analysis}

Data were collected and entered into a Microsoft Access database and were analyzed using SPSS (Statistical Package for the Social Science, version 20, IBM, and Armonk, New York). Continuous data was expressed in the form of mean \pm SD or median (range) while nominal data was expressed in form of frequency (percentage). Chisquared test was used to compare the nominal data of different groups in the study while Student's $t$ test was used to compare the mean of different two groups. ROC curve was used to determine the diagnostic accuracy of the PC method for the measurement of EF. $P$ value was considered significant if $<0.05$. 

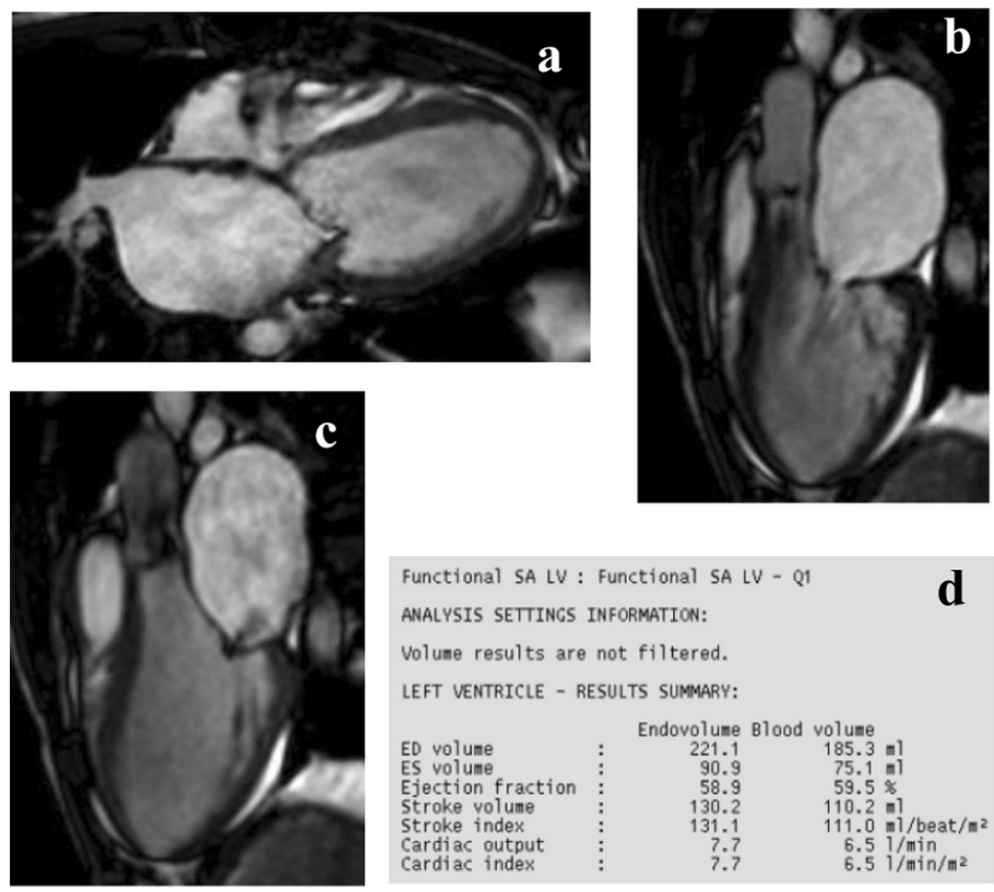

Fig. 6 A 26-year-old male patient with rheumatic valvular heart disease presented by dyspnea grade III. The performed CMR showed mitral and aortic regurge. a Aortic regurge is seen in a three-chamber view during diastole. b, c Mitral regurge is seen in three and four-chamber views during systole. $\mathbf{d}$ The volumetric analysis shows that ejection fraction estimated by volumetric short-axis method (EFvol) was 59\% (normal)

\section{Results}

The demographic data showed that the mean age $( \pm$ SD) of the study group was $42.33 \pm 12.09$ years, and for the healthy group was $38.60 \pm 9.83$ years. The majority of the studied subjects were males in both groups. As regard sex and age, there were no significant differences between both groups $(P>0.05)$. The complaints of the patients of the study group were dyspnea, chest pain, and easy fatigability which were presented in $13(52 \%)$, $10(40 \%)$, and $2(8 \%)$ patients, respectively. The majority of patients (14 patients, 56\%) had rheumatic heart disease while dilated cardiomyopathy and ischemic heart disease in 6 (24\%) and 5 patients (20\%), respectively.

In our study, it was noticed that the frequency of aortic regurgitation was more than aortic stenosis; 12 patients (48\%) had variable degrees of aortic regurgitation while only 2 patients $(8 \%)$ had aortic stenosis. Combined regurgitation and stenosis was detected in 2 patients (8\%). The other 9 patients in the study (36\%) had a normal aortic valve. According to the mitral valve, mitral regurgitation was encountered more frequently in this study (11 cases, 44\%) than mitral stenosis (4 cases, 16\%). Patients with mitral regurgitation could be further classified into variable degrees of regurgitation including mild regurge (3 patients, $12 \%)$, moderate regurge $(4,16 \%)$, and marked regurge $(4,16 \%)$. All the 4 cases of mitral stenosis had a severe degree of stenosis. Combined regurgitation and stenosis were encountered in 3 patients (12\%). no abnormality could be detected in the mitral valve in the other 7 patients of the study (28\%).

Regarding assessment of the cardiac function and measurement of the $\mathrm{EF}$ by both volumetric and $\mathrm{PC}$ methods in the healthy group, according to the volumetric method, calculation of the mean EF in the study was $62.44 \pm 6.61$, while that calculated by the PC method was $64.34 \pm 5.33$ which was not significant $(P=0.62)$ showing the validity of the PC method. In the patients' group, according to the volumetric method, calculation of the mean EF was $47.17 \pm 14.31 \%$, which was significantly higher than that calculated by the PC method (29.39 \pm $7.98 \%)(P=0.02)$. Also, by the volumetric method, there were 18 patients $(72 \%)$ with impaired cardiac function and 7 (28\%) patients with normal function; while by the PC method, all the 25 patients of the study had impaired cardiac function. As shown in Table 2, the current study demonstrates the diagnostic accuracy of PC-CMR in diagnosing impaired cardiac function having $89.29 \%$ sensitivity and $85.7 \%$ specificity with the area under the curve of 0.87 ( $P=0.00$, Fig. 3).

Figures 4, 5, 6, 7, 8, and 9 show three illustrative cases with volumetric and PC-CMR analysis.

\section{Discussion}

The ejection fraction describes the volume of blood pumped out from the left ventricle per beat and is widely used as a rapid and rough indicator of left 

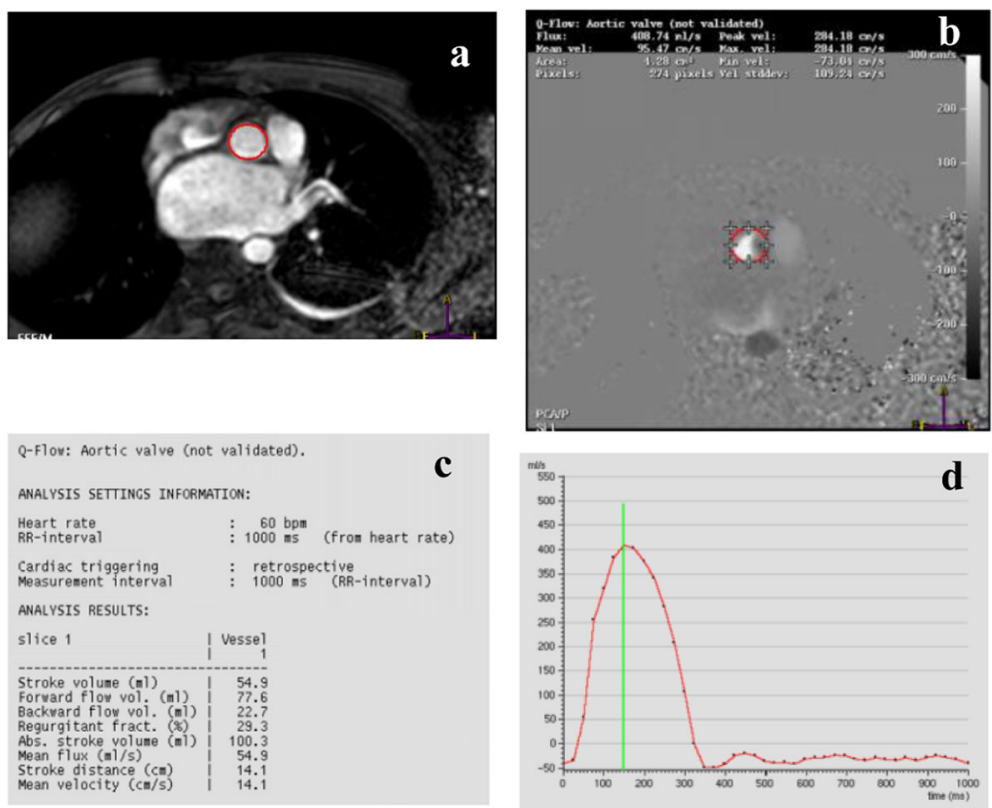

Fig. 7 On the other hand, the same patient using the phase-contrast analysis, the LV stroke volume (SVPC) was $54.9 \mathrm{ml}$ and the ejection fraction $(E F P C)$ was $(54.9 / 221.1) \times 100=24.8 \%$ (severe dysfunction). a The magnitute image showing the detailed anatomy, contour, and the shape of the aorta. $\mathbf{b}$ The phase velocity map depicts the velocity and direction of flow in each pixel within the aortic valve during systole. c Q-flow analysis of the aortic flow. $\mathbf{d}$ By outlining the contours of the aorta throughout each phase in the cardiac cycle, a flow curve can be generated to determine aortic forward reverse stroke volume and flow

ventricle contractile function. The calculation of LVEF has important diagnostic, prognostic, and therapeutic implications [25-28].

EF is widely used in the initial classification and risk stratification of patients with heart failure [29]. MRI is the preferred method for the estimation of left ventricular volume and ejection fraction in patients with heart failure [30]. The term "normal EF" is clinically interpreted as sufficient amount of blood pumped by the heart to cover the demands of different body organs.

The aim of this study was to compare cine volumetry and phase contrast as cardiac MRI techniques for estimation of the EF in patients with valvular insufficiency.
In this study, in the 10 healthy control subjects, the LVEF measured with the volumetric technique was equivalent to the EF measured by PC technique; therefore, in absence of valvular insufficiency, the EF can be reliably measured using both CMR techniques with minimal discordance.

On the other hand, among the 25 patients with valvular abnormalities, the volumetric CMR method had falsely assigned $28 \%$ of patients to have normal cardiac function, although they actually had clinical manifestations of heart failure. However, the LVEF calculated according to PC-CMR method showed that these patients had impaired cardiac function. Moreover, there was mismatching in the degree of cardiac function

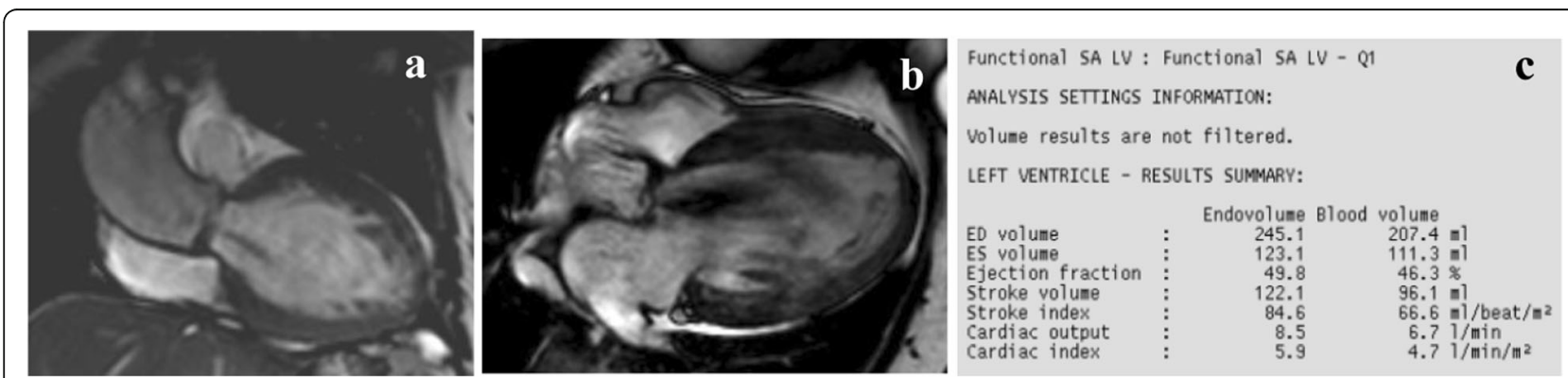

Fig. 8 A 55-year-old female patient with rheumatic valvular heart disease presented by dyspnea, chest pain, and lower limb edema. The performed CMR showed aortic regurge seen in a two-chamber view during diastole and $\mathbf{b}$ four-chamber view during diastole. c The volumetric analysis shows that ejection fraction estimated by volumetric short-axis method (EFvol) was $49.8 \%$ (mild dysfunction) 

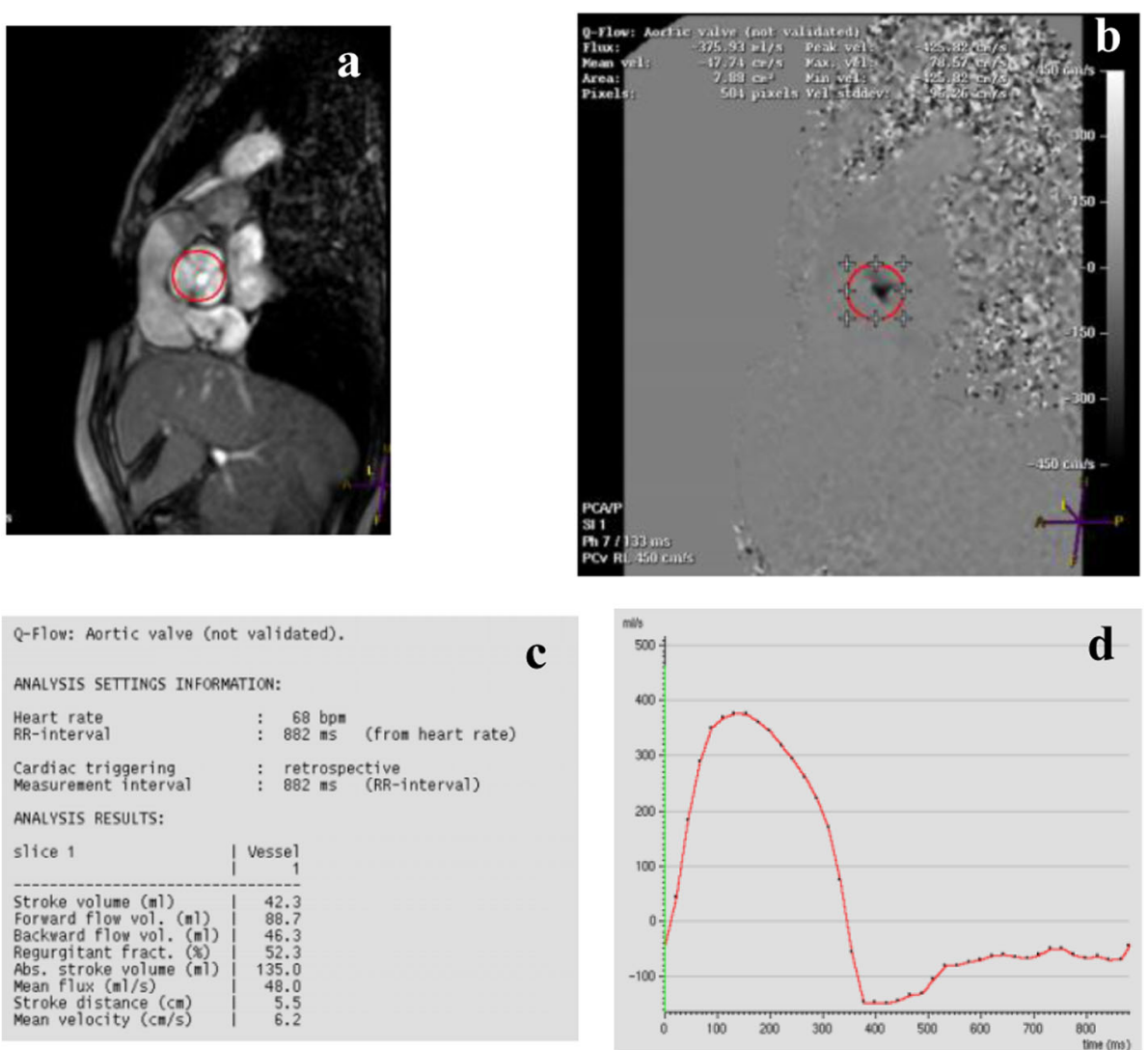

Fig. 9 On the other hand, the same patient using the phase-contrast analysis, the LV stroke volume (SVPC) was $42.3 \mathrm{ml}$ and ejection fraction (EFPC) was $(42.3 / 245.1) \times 100=17.3 \%$ (severe dysfunction). a The magnitute image showing the detailed anatomy, contour, and the shape of the aorta. $\mathbf{b}$ The phase velocity map depicts the velocity and direction of flow in each pixel within the aortic valve during systole. $\mathbf{c}$ Q-flow analysis of the aortic flow. $\mathbf{d}$ By outlining the contours of the aorta throughout each phase in the cardiac cycle, a flow curve can be generated to determine aortic forward reverse stroke volume and flow

impairment between the volumetric and PC methods of cardiac MRI in these patients.

The volumetric method for estimation of LVEF seems to be erroneous in the calculation of the true blood volume pumped into the aorta that actually determines the clinical condition of the patient and his prognosis.

In the presence of valvular regurgitation, when the left ventricle contracts, the end-diastolic blood volume is divided into three portions: the ejection fraction $\mathrm{EF}$, the regurgitant fraction, and the residual fraction (ESV); and each of these fractions has its relevant indicator. The EF indicates the efficacy by which the heart provides blood to different body organs; the regurgitant fraction indicates the severity of valve pathology, whereas the residual fraction represents the efficiency of cardiac muscle contraction that would in turn judge the extent of benefit that would be gained after surgical management.

Clancy et al. [31] hypothesized the term "forward EF" to describe the forward stroke volume divided by EDV and suggested that it may be a useful index of LV function, since it represents LV emptying into the aorta. Forward LVEF is a composite marker of valve regurgitation severity and LV systolic dysfunction. The "total" LVEF measured by Simpson's method may grossly underestimate the extent of intrinsic myocardial systolic dysfunction [32].

Dupuis et al. [33] stated that the forward LVEF (LV outflow tract SV divided by LVEDV) is superior to the total LVEF in predicting outcomes in mitral regurgitation. Other similar studies have described the use of PCCMR for calculation of the mitral regurgitant volume by subtraction of the aortic forward flow calculated at the aortic root from the total left ventricular stroke volume (LVSV) obtained from planimetry of the LV end-diastolic and end-systolic contours [34].

Accurate quantification of valve regurgitation is crucial for the optimal management of patients with valvular heart disease [35]. PC-CMR is a well-validated method for quantifying left-sided regurgitant valvular lesions [36, 37].

To the best of our knowledge, this is the first time to combine the cine volumetric CMR method with PC flow 
measurements for calculation of the left ventricular volumes and EF. The study shows that EF calculated by cine volumetry cannot be relied upon in the presence of valvular dysfunction, while PC-CMR is more accurate in this regard.

This study shows that cardiac MRI can accurately calculate LVEF in patients with valvular heart disease; however, certain limitations do exist in this work; a few of them could be addressed through further work, while others are inherent to the methodology we used.

1- Reference values from normal volunteers of racial and ethnic concerns with different age and sex groups are not established yet. The same also applies regarding patients' ranking for the degree of impaired cardiac function.

2- Phase-contrast velocity mapping can be affected by many technical factors such as translational valve motion, temporal blurring, partial volume degradation, and velocity offset errors due to eddy currents or magnetic field inhomogeneity, and so the technique needs to be standardized to validate the accuracy of its results [38].

For routine clinical utilization of the metrics obtained in this study, reference values from normal volunteers of racial and ethnic concerns need to be established. A parallel similar scale study is also needed in cardiac patients to determine the ranking for the degree of impaired cardiac function. Also PC calculation method of EF may be applied in further studies on other conditions expressing reverse in the cardiac hemodynamic-like ventricular septal defect where the blood pumped out of the LV is divided into two fractions: one fraction is ejected into the aorta whereas the other fraction is shunted to the low-pressure right ventricle and miscalculated as a part of LVEF.

\section{Conclusion}

Functional assessment of the left ventricle is important in the management planning of patients with valvular heart diseases. PC-CMR is accurate in calculation of the LVEF and assessment of left ventricular function in regurgitant valvular heart diseases, being superior to cine volumetry in this regard that would have an important impact on management of such patients.

\footnotetext{
Abbreviations

CMR: Cardiac magnetic resonance; EDV: End-diastolic volume; EF: Ejection fraction; ESV: End-systolic volume; LV: Left ventricular; LVEF: The left ventricular ejection fraction; LVOT: Left ventricular outflow tract; MRI: Magnetic resonance imaging; PC: Phase contrast; PC-CMR: Phasecontrast cardiac magnetic resonance; RHD: Rheumatic heart diseases; SV: Stroke volume; VHD: Valvular heart diseases
}

\section{Acknowledgements}

No acknowledgements

\section{Authors' contributions}

All authors have read and approved the manuscript. HAY, AESH, NAAM, and ASI contributed to the idea selection, data analysis, and research writing. $\mathrm{HAY}$ and ASI contributed to the study design. AESH and AE contributed to the case selection and collection of clinical data. AESH performed the MRI examination. HAY, NAAM, and ASI contributed to the data interpretation.

\section{Funding}

The authors declare that they did not receive any funding, grants, or financial support in this research work.

\section{Availability of data and materials}

The datasets generated and/or analyzed during the current study are not publicly available due to technical issues but are available from the corresponding author on reasonable request.

\section{Ethics approval and consent to participate}

The study has been approved by the Institutional Review Board and Ethical Comity of the Faculty of Medicine of Assiut University under the reference number 17100583. An informed written consent was obtained from all participants in the study.

\section{Consent for publication}

The corresponding author of the paper clearly states that all authors gave him clear acceptance to publish the paper. All patients included in this research gave written informed consent to publish the data contained within the study. Confidentiality of the patient data were kept throughout the whole study period. All the enclosed images in the manuscript are anonymous carrying no patient identity.

\section{Competing interests}

The authors declare that they have no competing interests.

\section{Author details}

${ }^{1}$ Radiology Department, Faculty of Medicine, Assiut University, Assiut, Egypt. ${ }^{2}$ Cardiothoracic Department, Faculty of Medicine, Assiut University, Assiut, Egypt. ${ }^{3}$ Radiology Department, Faculty of Medicine, Ain Shams University, Cairo, Egypt.

Received: 7 November 2019 Accepted: 29 December 2019

Published online: 13 January 2020

\section{References}

1. Curtis JP, Sokol SI, Wang Y, Rathore SS, Ko DT, Jadbabaie F et al (2003) The association of left ventricular ejection fraction, mortality, and cause of death in stable outpatients with heart failure. J Am Coll Cardiol 42(4):736-742

2. Knauth AL, Gauvreau K, Powell AJ, Landzberg MJ, Walsh EP, Lock JE et al (2008) Ventricular size and function assessed by cardiac MRI predict major adverse clinical outcomes late after tetralogy of Fallot repair. Heart. 94(2): 211-216

3. Piotr Ponikowski Voors AA, Anker SD, Cleland JGF, AJSC U, Harjola V, Germany VF et al (2016) 2016 ESC Guidelines for the diagnosis and treatment of acute and chronic heart failure The Task Force for the diagnosis and treatment of acute and chronic heart failure of the European Society of Cardiology ( ESC ) developed with the special contribution. Eur Heart J 128(20/may):1-85

4. Zange L, Muehlberg F, Blaszczyk E, Schwenke S, Traber J, Funk S et al (2019) Quantification in cardiovascular magnetic resonance: agreement of software from three different vendors on assessment of left ventricular function, 2D flow and parametric mapping 08 Information and Computing Sciences 0801 Artificial Intelligence and Image. J Cardiovasc Magn Reson 21(1):12

5. Grothues F, Smith GC, Moon JCC, Bellenger NG, Collins P, Klein HU et al (2002) Comparison of interstudy reproducibility of cardiovascular magnetic resonance with two-dimensional echocardiography in normal subjects and in patients with heart failure or left ventricular hypertrophy. Am J Cardiol 90(1):29-34

6. Hoffmann R, Von Bardeleben S, Ten Cate F, Borges AC, Kasprzak J, Firschke C et al (2005) Assessment of systolic left ventricular function: a multi-centre 
comparison of cineventriculography, cardiac magnetic resonance imaging unenhanced and contrast-enhanced echocardiography. Eur Heart J 26(6): 607-616

7. Joshi SB, Connelly KA, Jimenez-Juan L, Hansen M, Kirpalani A, Dorian P et al (2012) Potential clinical impact of cardiovascular magnetic resonance assessment of ejection fraction on eligibility for cardioverter defibrillator implantation. J Cardiovasc Magn Reson 14(1):69

8. Didier D (2003) Assessment of valve disease: qualitative and quantitative. Magn Reson Imaging Clin N Am 11(1):115-134

9. Markl M, Kilner PJ, Ebbers T (2011) Comprehensive 4D velocity mapping of the heart and great vessels by cardiovascular magnetic resonance. Cardiovasc Magn Reson 13:7

10. Nayak KS, Nielsen JF, Bernstein MA, Markl M, Gatehouse PD, Botnar RM et al (2015) Cardiovascular magnetic resonance phase contrast imaging. J Cardiovasc Magn Reson 17:71

11. Gelfand EV, Hughes S, Hauser TH, Yeon SB, Goepfert L, Kissinger KV et al (2006) Severity of mitral and aortic regurgitation as assessed by cardiovascular magnetic resonance: optimizing correlation with Doppler echocardiography. J Cardiovasc Magn Reson 8(3):503-507

12. Hundley WG, Li HF, Willard JE, Landau C, Lange RA, Meshack BM et al (1995) Magnetic resonance imaging assessment of the severity of mitral regurgitation: comparison with invasive techniques. Circulation. 92(5):1151-1158

13. Kilner PJ, Gatehouse PD, Firmin DN (2007) Flow measurement by magnetic resonance: a unique asset worth optimising. J Cardiovasc Magn Reson 9: $723-728$

14. Kon MWS, Myerson SG, Moat NE, Pennell DJ (2004) Quantification of regurgitant fraction in mitral regurgitation by cardiovascular magnetic resonance: comparison of techniques. J Heart Valve Dis 13(4):600-607

15. Maganti K, Rigolin VH, Sarano ME, Bonow RO (2010) Valvular heart disease: diagnosis and management. Mayo Clinic Proceedings. Elsevier Ltd, New York, pp 483-500

16. Nishimura RA, Otto CM, Bonow RO, Carabello BA, Erwin JP, Fleisher LA et al (2017) 2017 AHA/ACC focused update of the 2014 AHA/ACC guideline for the management of patients with valvular heart disease: a report of the American College of Cardiology/American Heart Association task force on clinical practice guidelines. J Am Coll Cardiol 70(2):252-289

17. Vahanian A, Alfieri $O$, Andreotti F, Antunes MJ, Barón-Esquivias G, Baumgartner $\mathrm{H}$ et al (2012) Guidelines on the management of valvular heart disease (version 2012). Eur Heart J 33(19):2451-2496

18. Quinones MA, Waggoner AD, Reduto LA, Nelson JG, Young JB, Winters WL et al (1981) A new simplified and accurate method for determining ejection fraction with two-dimensional echocardiography. Circulation. 64(4):744-753

19. Foley TA, Mankad SV, Anavekar NS, Bonnichsen CR, Morris MF, Miller TD et al (2012) Measuring left ventricular ejection fraction-techniques and potential pitfalls. Eur Cardiol 8:108-114

20. Wisneski JA, Pfeil CN, Wyse DG, Mitchell R, Rahimtoola SH, Gertz EW (1981) Left ventricular ejection fraction calculated from volumes and areas: underestimation by area method. Circulation. 63(1):149-151

21. Kamperidis V, Marsan NA, Delgado V, Bax JJ (2016) Left ventricular systolic function assessment in secondary mitral regurgitation: left ventricular ejection fraction vs. speckle tracking global longitudinal strain. Eur Heart J 37(10):811-816

22. Gatehouse PD, Keegan J, Crowe LA, Masood S, Mohiaddin RH, Kreitner KF et al (2005) Applications of phase-contrast flow and velocity imaging in cardiovascular MRI. Eur Radiol 15:2172-2184

23. Ko SM, Song MG, Hwang HK (2012) Evaluation of the aortic and mitral valves with cardiac computed tomography and cardiac magnetic resonance imaging. Int J Cardiovasc Imaging 28(2 SUPPL):109-127

24. Lang RM, Badano LP, Mor-Avi V, Afilalo J, Armstrong A, Ernande L et a (2015) Recommendations for cardiac chamber quantification by echocardiography in adults: an update from the American society of echocardiography and the European association of cardiovascular imaging. Eur Heart J Cardiovasc Imaging 16(3):233-271

25. Taylor GJ, Humphries JO, Mellits ED (1980) Predictors of clinical course, coronary anatomy and left ventricular function after recovery from acute myocardial infarction. Circulation. 62(5):960-970

26. Shaw LJ, Peterson ED, Kesler K, Hasselblad V, Califf RM (1996) A metaanalysis of predischarge risk stratification after acute myocardial infarction with stress electrocardiographic, myocardial perfusion, and ventricular function imaging. Am J Cardiol 78(12):1327-1337
27. Asferg C, Usinger L, Kristensen TS, Abdulla J (2012) Accuracy of multi-slice computed tomography for measurement of left ventricular ejection fraction compared with cardiac magnetic resonance imaging and two-dimensional transthoracic echocardiography: a systematic review and meta-analysis. Eur J Radiol 81(5):e757-e762

28. White HD, Norris RM, Brown MA, Brandt PW, Whitlock RM, Wild CJ (1987) Left ventricular end-systolic volume as the major determinant of survival after recovery from myocardial infarction. Circulation. 76(1):44-51

29. Raman SV, Simonetti OP (2009) The CMR examination in heart failure. Heart Fail Clin 5(3):283-300

30. Bellenger NG, Burgess MI, Ray SG, Lahiri A, Coats AJS, Cleland JGF et al (2000) Comparison of left ventricular ejection fraction and volumes in heart failure by echocardiography, radionuclide ventriculography and cardiovascular magnetic resonance. Are they interchangeable? Eur Heart J 21(16):1387-1396

31. Clancy KF, Hakki AH, Iskandrian AS, Hadjimiltiades S, Mundth ED, Hakki AH et al (1985) Forward ejection fraction: a new index of left ventricular function in mitral regurgitation. Am Heart J 110(3):658-664

32. Gaasch WH, John RM, Aurigemma GP (1995) Managing asymptomatic patients with chronic mitral regurgitation. Chest 108:842-847

33. Dupuis M, Mahjoub H, Clavel MA, Côté N, Toubal O, Tastet L et al (2017) Forward left ventricular ejection fraction: a simple risk marker in patients with primary mitral regurgitation. J Am Heart Assoc 6(11):e006309

34. Lopez-Mattei JC, Shah DJ (2013) The role of cardiac magnetic resonance in valvular heart disease. Methodist Debakey Cardiovasc J 9(3):142-148

35. Nkomo VT, Gardin JM, Skelton TN, Gottdiener JS, Scott CG, Enriquez-Sarano M (2006) Burden of valvular heart diseases: a population-based study. Lancet. 368(9540):1005-1011

36. Westenberg JJM, Doornbos J, Versteegh MIM, Bax JJ, Van Der Geest RJ, De Roos A et al (2005) Accurate quantitation of regurgitant volume with MRI in patients selected for mitral valve repair. Eur J Cardio-thoracic Surg 27(3): 462-467

37. Aquaro GD, Barison A, Todiere G, Festa P, Ait-Ali L, Lombardi M et al (2015) Cardiac magnetic resonance "virtual catheterization" for the quantification of valvular regurgitations and cardiac shunt. J Cardiovasc Med 16(10):663-670

38. Chernobelsky A, Shubayev O, Comeau CR, Wolff SD (2007) Baseline correction of phase contrast images improves quantification of blood flow in the great vessels. J Cardiovasc Magn Reson 9(4):681-685

\section{Publisher's Note}

Springer Nature remains neutral with regard to jurisdictional claims in published maps and institutional affiliations.

\section{Submit your manuscript to a SpringerOpen ${ }^{\circ}$ journal and benefit from:}

- Convenient online submission

- Rigorous peer review

- Open access: articles freely available online

High visibility within the field

- Retaining the copyright to your article

Submit your next manuscript at $>$ springeropen.com 